\title{
Influence of Different Copolymer Based Compatibilizers on Performance of Pristine and Recycled PP/PE blends
}

\author{
C. Rosales ${ }^{1}$, A. Costantino', G. Palazzo ${ }^{2}$, C. Bernal ${ }^{3}$, R. Defacio Dutra ${ }^{4}$, V. Pettarin ${ }^{1, * *}$ \\ ${ }^{1}$ Institute of Materials Science and Technology (INTEMA), University of Mar del Plata/CONICET, Mar del Plata, Argentina \\ ${ }^{2}$ AMPACET Latin America, Buenos Aires, Argentina \\ ${ }^{3}$ Institute of Technology in Polymers and Nanocomposites (ITPN), University of Buenos Aires/CONICET, Buenos Aires, \\ Argentina \\ ${ }^{4}$ AMPACET Latin America, São Paulo, Brasil
}

*Corresponding author: E-mail: pettarin@fi.mdp.edu.ar; Tel.: +54-223-4816600

DOI: $10.5185 /$ amlett.2020.111572

One of the big engineering challenges in recycling thermoplastics is to manufacture competitive products in terms of mechanical properties such as toughness or strength. This process by which recycled waste is transformed into materials with improved performance, adding value and widening their application field, is known as upcycling. One of the strategies to improve PE/PP blends performance is to add a special compatibilizer, and this is the aim of the present work. To achieve this objective, four different commercial masterbatch compatibilizers based on copolymers were added to pristine blends. The compatibilizer that induced the best performance in virgin blends was selected to be applied in post-consumer recycled (PCR) blends, after minimizing its dosage to diminish costs. All blends were prepared simulating industrial processing conditions. Microstructure and morphology of blends were analyzed by DSC and SEM. Tensile and fracture studies were carried out at quasi-static loading conditions. Fracture surfaces were studied by SEM. It was observed that results found for pristine blends were consistent with those of recycled blends, and the addition of compatibilizing masterbatches improved the performance of both pristine and recycled PP/PE blends, in spite of the unpredictable composition and the presence of impurities of PCR blends.

\section{Introduction}

Plastic waste accumulation is one of the main environmental problems of the XXI century. Plastics could be seriously harmful for environment due to their slow and complex degradation process. Polymers contained in urban solid waste streams are mainly (nearly 60\%) polyethylene (PE) and polypropylene (PP), with other commercial polymers such as polystyrene (PS) and polymethylmethacrylate (PMMA) in smaller proportions. Individual separation of these plastic wastes is usually complicated and not economically acceptable. However, in Latin America, the infrastructure and technology necessary to separate PE/PP from the rest of the plastic waste stream at low cost at an industrial levels is available, and then $\mathrm{PE} / \mathrm{PP}$ blends seems a technically and economically viable recycling alternative. In fact, a large amount of work has been focused on the study of mechanical properties of mixed plastics, but it was mostly applied on virgin materials [1-6].

The difficult task of dealing with blends is to obtain a material with good mechanical properties, since the presence of a polymer dispersed in a second one may strongly change these properties and limit blends use in conventional applications. The problem is emphasized in the case of recycled blends which suffer irreversible thermo-oxidative degradation during successive manufacturing processes, i.e., these recycled blends usually present lower thermal and mechanical properties compared to virgin ones [7]. It is then evident that one of the big engineering challenges of recycling thermoplastics is to manufacture competitive pieces or products in terms of mechanical properties - such as toughness or strength -to widen their application field. An upcycling of the inseparable mixtures for value-added goals remains a challenge for the scientific community [8].

In particular, in the case of PE/PP blends, their marked incompatibility is well known as it has been previously demonstrated [9], resulting detrimental to their mechanical and thermal performance. However, it is also known that it is possible to obtain higher quality virgin and recycled blends when compatibilizing copolymers are used $[4,10]$. Unfortunately, and based on referenced literature and on sustainability considerations, it can be concluded that a universal compatibilizing agent cannot be cost-effective in every system/blend. Moreover, an analysis based on each specific blend (and its quality), requires a taylor-made compatibilizing agent/system, not only from a formulation standpoint but also technology-wise. And at this point is where masterbatches appear as a solution. Hybrid organicinorganic concentrates such as titanium dioxide in a polyethylene matrix are supplied commercially to the plastic industry as "white masterbatch". Masterbatch producers, are materials technology solutions providers and 


\section{Advanced Materials Letters www.vbripress.com/aml}

have been a part of the plastics industry since its early beginnings. Masterbatch companies deal with the effective and efficient combination of inorganic and organic materials, observing health and safety concerns. It is a common practice within the plastic industry to purchase pigment and additive concentrates in pellet form instead of powder, liquid or semisolid raw materials in order to produce high performance plastic parts. A low distribution and/or dispersion of organic or inorganic fillers or additives in a polymeric matrix can lead to poor final (optical, mechanical, etc.) properties. Good interaction between polymer phases leads to desirable mechanical performance because of the improved stress transfer between the phases [11], and particularly in PE/PP blends, interaction could be improved adding copolymers of the same chemical nature. Besides, copolymers are an effective and economical way of improving blend immiscibility $[\mathbf{1 2 , 1 3}]$. It is therefore clear that it is necessary to consider several approaches when dealing with PCR blends. The Masterbatch industry can and usually is the materials technology partner that helps PCR intensive industries process their raw materials into added value products.

It is also important at this point to recall that most studies deal with pristine or Post Industrial Recycled (PIR) materials. A typical example of this is observed in the work of Kallel et. al., who studied the effectiveness of low-cost commercial compatibilizers combined with several processes for two types of plastic blends: high-density polyethylene (HDPE)/polypropylene (PP) and high-density polyethylene/ polystyrene blends (PS), to gain insight into the recycling of wastes from those frequently encountered mixed plastics. However, they used virgin commercially available grades of HDPE, PP and PS [4].

In addition, most of works which deal with recycled blends evaluate mechanical performance by means of conventional mechanical tests such as tensile, flexural or non-instrumented impact tests which in many cases are not representative of actual loading situations [14-17]. In fact, Izod and Charpy tests have lost favour in engineering design because they cannot be used directly in design calculations. In their desire to characterize toughness of ductile polymers, many researchers have turned to fracture mechanics. Fracture tests that imply a more severe loading condition, i.e., load triaxiality, are more adequate to evaluate mechanical integrity of final pieces [18].

In this work, a complete morphological and mechanical evaluation of the effectiveness of different kinds of compatibilizers in PE/PP virgin and post consume recycled (PCR) blends is presented. The effect of commercially available copolymers and terpolymers as compatibilizers of $\mathrm{PE} / \mathrm{PP}$ blends was analyzed in comparison with a new compatibilizing approach in masterbatch form. One of the main objectives of this work is to evaluate the reliability extrapolation of data obtained on virgin polymer blends to actual PCR blends. The use of PCR blends - with not exactly known composition and presence of impurities - makes this study relevant since it implies a real case of study in collaboration with local companies that are representative of the plastic industry landscape in Latin America. Such industrial environment poses a challenge rarely faced by individual laboratory research groups. Local commercial availability of materials is key in order to provide for compatibilization of PCR blends with ever-variable composition. Moreover, the use of fracture mechanics to assess differences in microstructure induced by the presence of $\mathrm{MB}$, enhances the novelty of this work.

\section{Experimental}

\section{Materials and blends preparation}

The virgin and recycled polymer blends were supplied by Ampacet Latin America. Five pristine blends were studied: LLDPE/PP 50/50\% w/w without any compatibilizer (PR-0) and LLDPE/PP 45/45\% w/w with $10 \% \mathrm{w} / \mathrm{w}$ of four different masterbatches (MB). 50/50\% w/w composition was selected based on preliminary studies of recycled blends, with the aim of compare pristine and recycled blends behavior. The blends under investigation included as compatibilizer block (PR-10MB1), random (PR10MB2), and ter (PR-10MB3) PE-PP copolymers (2630PC from Cuyolen, RP347 from Braskem, and Symbios 4102 from Braskem, respectively); and a compatibilizer commercialized as 1000889-S (Ampacet) for non-polar PCR blends (PR-10MB4). The distinctive characteristic of MB-4 is that instead of being based on a PE-PP copolymer, the main resin is PE, and includes a stearates/amides and waxes blend (Struktol TR052), and a low content of an antioxidant to protect the polymers during processing. A blend with $3 \% \mathrm{w} / \mathrm{w}$ of this last compatibilizer was also prepared (PR-3MB4). LLDPE/PP blends (with and without compatibilizers) were processed in a single screw extruder with vent LabTech LE25-30/CV-HA (high-grade nitride steel screw and barrel, screw diameter $25 \mathrm{~mm}$ and L/D ratio of 30), similar to industrial processing conditions, with temperatures from hopper to die varying between 190 and $240^{\circ} \mathrm{C}$.

The obtained blends were then compression molded in a hydraulic press at $210^{\circ} \mathrm{C}$, under $1 \mathrm{MPa}$ for $15 \mathrm{~min}$ followed by $10 \mathrm{MPa}$ for $8 \mathrm{~min}$. Plaques $(150 \mathrm{~mm}$ x $200 \mathrm{~mm}$ x $2 \mathrm{~mm}$ ) were allowed to cool slowly in order to avoid thermal residual stresses. Samples for different tests were mechanically machined from plaques using a drill.

Also, two 50/50 post-consumer recycled blends were analyzed: LLDPE/PP with no compatibilizer (PCR-0) and with $3 \% \mathrm{w} / \mathrm{w}$ of the compatibilizer with best performance in the pristine blends, 1000889-S (PCR-3MB4). Recycled $\mathrm{PE} / \mathrm{PP}$ blend was obtained from separation of postconsumer waste streams, followed by reprocessing and pelletization in a Erema recycling machine (Fig. 1). Even though the machine used to obtain virgin blends is different from that used to obtain PCR blends, comparison is only performed between blends of the same kind, i.e. pristine blends are not compared with recycled blends. PCR blend composition is unpredictable due to its origin and the presence of impurities, as seen by optical microscopy 


\section{Advanced Materials Letters www.vbripress.com/aml}

(Fig. 1). In addition, one of the greatest challenges in recycling engineering of thermoplastics is to manufacture materials or products that are competitive with the virgin ones, both in performance and cost. It is therefore crucial to minimize recycling steps to minimize costs. The quantitative description of each PCR blend requires many resources and time, which in turn may hardly increase material's cost. That is the reason why it is more important to know blend nature than blend specific composition, being this more representative of the actual industrial situation. Then PCR blends were processed following the same industrial compression conditions used for pristine blends.

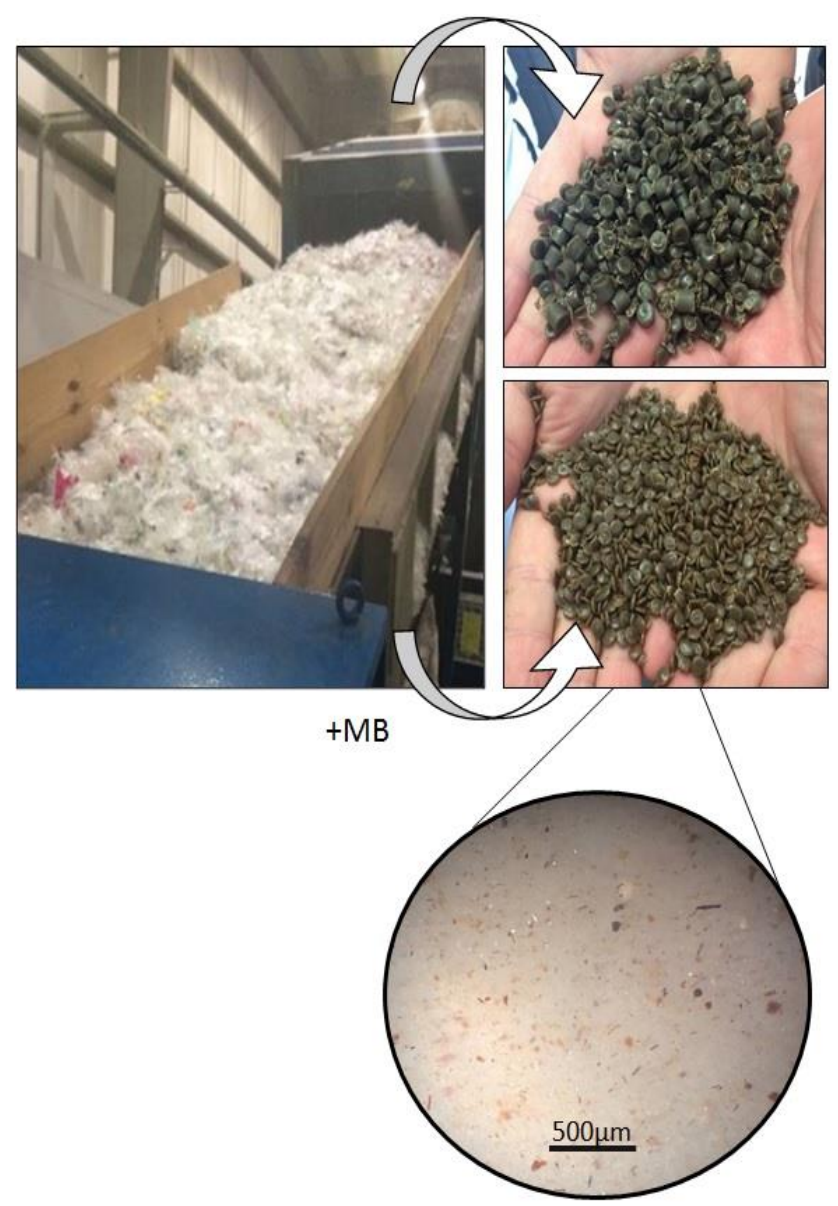

Fig. 1. Stages of recycling process of LLDPE/PP post-consumer (PCR) recycled blends and their impurities as observed by optical microscopy.

\section{Microstructure and morphology characterization}

Thermal behaviour of LLDPE/PP blends were analyzed by means of Differential Scanning Calorimetry (DSC) carried out with a Pyris 1 Perkin Elmer under nitrogen atmosphere. Samples were heated from 50 to $200^{\circ} \mathrm{C}$ at $10^{\circ} \mathrm{C} / \mathrm{min}$. Crystallinity of LLDPE and PP in blends were calculated using the following relationship:

$$
x_{c}(\%)=\frac{\left(\Delta H_{f}^{o b s} / \Delta H_{f}^{0}\right)}{\Phi} 100
$$

where $\Delta H_{f}^{o b s}$ is the observed heat of fusion values, $\Delta H_{f}^{0}$ is the heat of fusion value for $100 \%$ crystalline LLDPE
$(293 \mathrm{~J} / \mathrm{g})[\mathbf{1 9}]$ or PP $(207 \mathrm{~J} / \mathrm{g})$ [20], and $\Phi$ is the weight fraction of the constituent. In order to evaluate the influence of processing in crystallinity, it was evaluated from data of the first heat in DSC.

To reveal morphology, micrographs were taken with a JSM 6460 scanning electron microscope (SEM). Samples were notched, frozen in liquid nitrogen and cryo-fractured, and then coated with a thin gold layer, prior to observation. XRD analysis was performed using a Phillips X'PERT MPD diffractometer in reflection mode $(\mathrm{Cu} \mathrm{K} \alpha$ radiation $\lambda=1.5418^{\circ} \mathrm{A}$, generator voltage $40 \mathrm{kV}$, current $40 \mathrm{~mA}$, sample to detector distance $240 \mathrm{~mm}$ ) to observe the blends structure.

Dynamic oscillatory shear rheological behaviour was evaluated by using an Anton Paar, Physica MCR-301 instrument in parallel-plate geometry with a diameter of $25 \mathrm{~mm}$ at $210^{\circ} \mathrm{C}$. Measurements were performed with a shear stress amplitude, of $0.1 \%$ and frequency range of $0.01-100$.

\section{Mechanical performance}

Mechanical performance was assessed by means of tensile and fracture in mode I tests in a universal testing machine (Instron Model 5982). Five specimens of each blend were tested for both mechanical test.

Tensile tests were carried out on dog-bone specimens cut from moulded plaques, following ASTM D638 standard recommendations. Data for yield strength and Young's modulus were obtained from tests carried out at a crosshead speed of $5 \mathrm{~mm} / \mathrm{min}$ at room temperature.

Fracture tests were performed on mode I double edgenotched tensile specimens (DENT) at quasi-static loading conditions $(2 \mathrm{~mm} / \mathrm{min})$. DENT samples (nominal width $\mathrm{W}=30 \mathrm{~mm}$ and nominal length $\mathrm{S}=70 \mathrm{~mm}$ ) were cut from the mouldings. Sharp notches were introduced by scalpelsliding a razor blade having an on-edge tip radius of $13 \mu \mathrm{m}$ with a Ceast Notchvis notching machine, with a notch to width ratio $\mathrm{a} / \mathrm{W}=0.5$

The J-integral is conventionally defined for non-linear elastic materials as a path independent line integral. In fact, the single-specimen $\mathbf{J}$ formulation has been used in the past to characterize ductile fracture in polymers [21]. The J-integral approach is a natural extension of linear elastic fracture mechanics and works best for not too ductile fractures. It was chosen in this work as the Fracture Mechanics methodology to evaluate blends fracture toughness. The value of the $\mathrm{J}$ integral was computed from the following expression:

$$
J=\frac{\eta U}{B(W-a)}
$$

The fracture energy $U$ was calculated by integration of the load-displacement curves up to the maximum load point $\left(J_{\max }\right.$ parameter determination), and the factor shape $\eta$ for DENT samples is given by:

$$
\eta=-0.06+5.99 \frac{a}{w}-7.42\left(\frac{a}{w}\right)^{2}+3.29\left(\frac{a}{w}\right)^{3}
$$

Finally, fracture surfaces were studied by SEM. 


\section{Advanced Materials Letters www.vbripress.com/aml}

\section{Results and discussion}

\section{Pristine PP/LLDPE blends}

Fig. 2 displays the phase morphologies of cryo-fractured pristine LLDPE/PP blends. All blends exhibit a similar morphology characterized by a polypropylene matrix with islands of polyethylene which contain occlusions of the matrix material, as a typical "salami" structure [22]. However, a change in domains shape is observed from the addition of compatibilizers. While the blend without compatibilizer (PR-0) presents mainly spherical domains, the blend PR-10MB1 exhibits inclusions with an elliptically shaped structure, and the PR-10MB2, PR10MB3 and PR-10MB4 blends show a co-continuous phase structure. As reported by several authors [23-26], polyolefin blends are immiscible and incompatible. For incompatible systems like PR-0, interfacial tension is high, i.e., spherical droplets are mainly generated during cooling, minimizing surface energy. As compatibilization increases, contact area also increases, leading to different shapes of second phase inclusions, i.e. smaller particles, ellipses and co-continuous structures. Another distinctive characteristic is the adhesion between phases $[\mathbf{2 3 , 2 7 , 2 8}]$. In the case of PR-0 and PR-10MB1, dark zones (voids) indicative of unbounded phases are clearly seen, while no evidences of detachment between phases are observed in PR-10MB2, PR-10MB3 and PR-10MB4.

Curves corresponding to microstructural characterization of virgin blends are presented in Fig. 3 . Crystallinities calculated from DSC heating thermograms are detailed in Table 1. Pristine blends show two melting endotherms corresponding to PP and LLDPE phases in all cases. The melting points ( $\left.\mathrm{T}_{\mathrm{m}}\right)$ of PP and LLDPE are 160 and $125^{\circ} \mathrm{C}$ respectively, and the blending process has no significant effect on these points. This also shows that the two polymers are highly incompatible and the blend between PP and LLDPE is immiscible, providing another clear evidence of phase separation between PP and LLDPE $[14,15,29]$. It is noticeable the change in the shape of the melting peak of PP component for PR-10MB4 blend, being an indication of changes in PP crystallinity morphology, maybe the presence of a different PP phase. Crystallinity of both PP and LLDPE remains similar for all blends except in the case of PR-10MB4 in which PP crystallinity decreases while LLDPE crystallinity increases (Table 1). This is probably because MB4 is mainly based on a PE resin. XRD diffractograms of all virgin samples exhibit the characteristic diffraction peaks of the $\alpha$-phase of PP ( $2 \theta$ of 14.2 (110), 17.1 (040), 18.6 (130)), and also the characteristic crystalline peaks of orthorhombic crystals of LLDPE (2 $\theta$ of $21.4(110)$ and 23.7 (200)) [30,31]. It is clear from patterns evaluation that compatibilizers do not affect crystalline structure of constituents, as it was previously reported by other authors for polyethylene-polypropylene blends $[\mathbf{1 , 1 5}$. However, a shift of all peaks is observed in the presence of MB4 (see Table 2), indicating some changes in crystalline phases. Regarding melt rheological performance, it is known to be a good measure of the interfacial tension in blends and the compatibilizer performance. Blends in the molten state may be considered as heterogeneous solutions and compatibilizers may act as emulsifiers preventing agglomeration of the dispersed phase [4]. Indeed, the compatibilizers increase the interactions between components. Then, when an effective compatibilizer is added, the viscosity of a blend is expected to increase. In our blends, the presence of 10MB4 increased the blend viscosity, indicating that this compatibilizer improves the interaction between components.

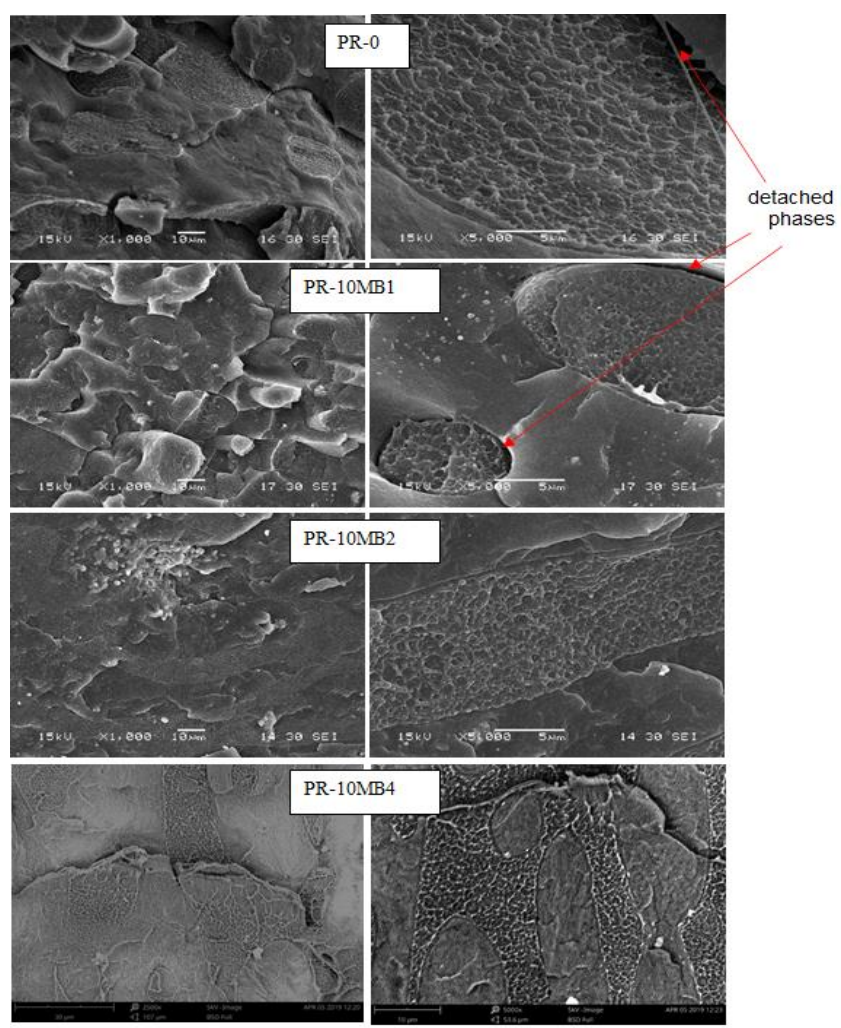

Fig. 2. Morphology of virgin blends as observed by scanning electron microscopy (left: low magnification level, right: high magnification level).

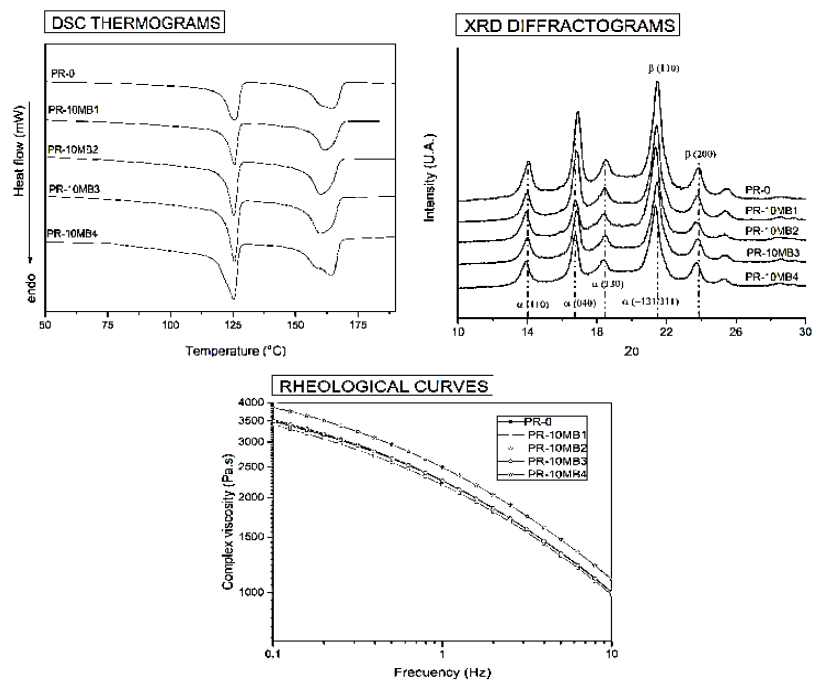

Fig. 3. Curves resulting from microstructural characterization of virgin blends. 


\section{Advanced Materials Letters www.vbripress.com/aml}

Table 1. Comparison of properties of pristine blend.

\begin{tabular}{lccccc}
\hline Sample & \multicolumn{2}{c}{ Crystallinity (\%) } & $\begin{array}{c}\text { Young's } \\
\text { Modulus } \\
(\mathbf{M P a})\end{array}$ & $\begin{array}{c}\text { Yield } \\
\text { stress } \\
(\mathbf{M P a})\end{array}$ & $\begin{array}{c}\mathbf{J}_{\max } \\
\left(\mathbf{K J} / \mathbf{m}^{2}\right)\end{array}$ \\
\hline PR-0 & LLDPE & $25 \pm 4$ & $706 \pm 58$ & $15.4 \pm 0.6$ & $17.3 \pm 4.6$ \\
& PP & $37 \pm 1$ & & & \\
PR-10MB1 & LLDPE & $26 \pm 4$ & $766 \pm 78$ & $13.2 \pm 1.0$ & $14.0 \pm 2.1$ \\
& PP & $39 \pm 1$ & & & \\
PR-10MB2 & LLDPE & $27 \pm 4$ & $813 \pm 65$ & $14.7 \pm 1.2$ & $15.8 \pm 3.9$ \\
& PP & $37 \pm 2$ & & & \\
PR-10MB3 & LLDPE & $26 \pm 4$ & $910 \pm 45$ & $16.1 \pm 0.7$ & $16.8 \pm 4.8$ \\
& PP & $35 \pm 2$ & & & \\
PR-10MB4 & LLDPE & $35 \pm 1$ & $788 \pm 65$ & $16.0 \pm 0.8$ & $26.3 \pm 2.8$ \\
& PP & $31 \pm 1$ & & & \\
PR-3MB4 & LLDPE & $30 \pm 1$ & $798 \pm 55$ & $15.2 \pm 0.5$ & $22.1 \pm 2.0$ \\
\hline
\end{tabular}

Table 2. XRD peaks corresponding to PP and PE crystalline phases.

\begin{tabular}{lccccc}
\hline \multirow{3}{*}{ Sample } & \multicolumn{5}{c}{ 20 in XRD diffractogram $\left(^{\circ}\right)$} \\
\cline { 2 - 6 } & \multicolumn{3}{c}{ PP crystalline phase } & PE crystalline phase \\
\cline { 2 - 6 } & $\alpha(110)$ & $\alpha(040)$ & $\alpha(130)$ & $\beta(110)$ & $\beta(200)$ \\
\hline PR-0 & 14.05 & 16.90 & 18.50 & 21.45 & 23.85 \\
PR-10MB1 & 13.98 & 16.78 & 18.44 & 21.44 & 23.78 \\
PR-10MB2 & 13.97 & 16.80 & 18.40 & 21.40 & 23.78 \\
PR-10MB3 & 13.98 & 16.83 & 18.48 & 21.43 & 23.84 \\
PR-10MB4 & 13.90 & 16.75 & 18.30 & 21.35 & 23.70 \\
PCR-0 & 14.16 & 17.06 & 18.71 & 21.66 & 24.01 \\
PCR-3MB4 & 14.10 & 17.00 & 18.65 & 21.60 & 23.92 \\
\hline
\end{tabular}

Mechanical and fracture results are listened in Table 1. Yield stress $\left(\sigma_{y}\right)$ is diminished by the presence of MB1 and MB2, while MB3 and MB4 have no significant effect on this property. Blends Young's modulus (E) is slightly increased by MB2 and MB3, while MB1 and MB4 have no influence on $\mathrm{E}$. It is then clear that it is difficult to make a statement about which would be the best compatibilizer only from tensile tests results. On the other side, fracture mechanics has been shown to be more sensitive to detect microstructural and morphological differences.

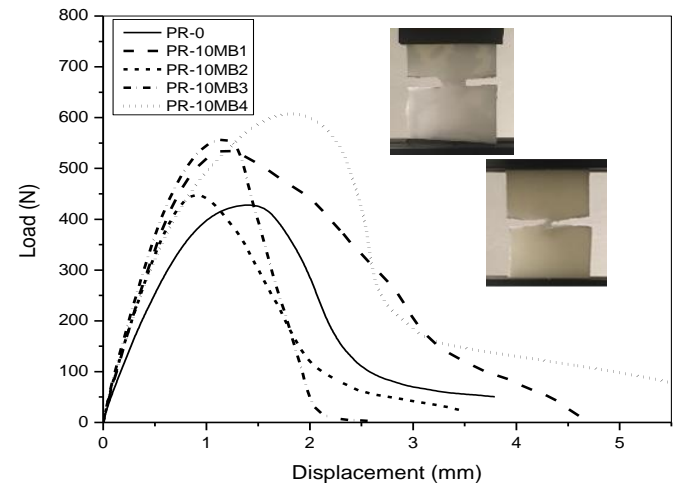

Fig. 4. Mechanical characterization of pristine blends. Load-displacement curves presented by pristine blends in fracture tests along with macroscopic damage.

Typical fracture load-displacement curves are presented in Fig. 4. All pristine blends show a stable fracture behaviour with crack tip blunting and specimen whitening. Furthermore, a crack path deviation is seen in the majority of samples: the crack does not develop in the plane that contains the initial sharp notch, i.e. the crack path is not controlled by the initial notch. It seems that the crack deviates its path when meets alternative ways, maybe located in weaker places in the boundaries between phases [32]. Besides, in some cases there is a competition between a stable crack propagation and ligament stretching (see inserted pictures in Fig. 4), this being the cause of standard deviation of fracture values depicted in Table 1. $\mathbf{J}_{\max }$ seems to be the same for all virgin modified blends with the exception of PR-10MB4 which presents the highest $\mathbf{J}_{\max }$ value, and hence a greatly improved fracture initiation resistance. When fracture surfaces are observed, it is seen that those of PR-0 and PR-MB1 blends exhibit ductile behavior with massive fibrillation (Fig. 5). On the other
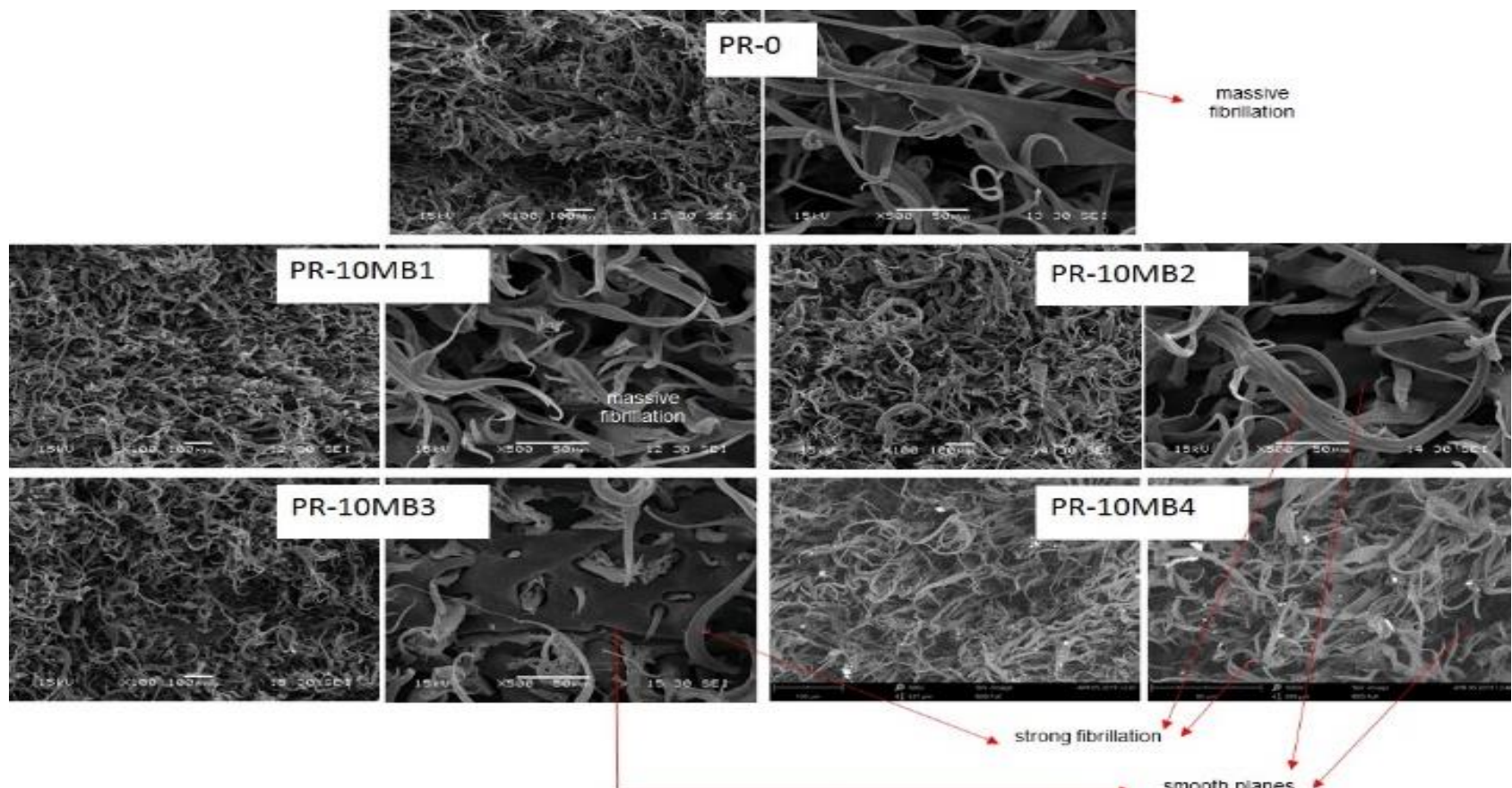

- strong fibrillation

$\rightarrow$

Fig. 5. SEM micrographs of fracture surfaces of pristine blends. 


\section{Advanced Materials Letters www.vbripress.com/aml}

hand, PR-MB2, PR-10MB3 and PR-10MB4 blends fractured surfaces show two different mechanisms: a ductile one characterized by a strong fibrillation and a brittle one characterized by smooth planes. These two mechanisms are competing during fracture leading to unstable crack propagation.

According to the obtained results, the compatibilized pristine blend that presents the best global performance is PR-10MB4, i.e., an improved fracture behavior without compromising conventional tensile properties. The main difference between MB4 and the other MBs is that MB4 includes low molecular weight components, which probably are located at the PP/PE interphases and act both as lubricants and dispersants.

However, one of the disadvantages of using compatibilizers is their relatively high cost and hence, their content must be maintained as lowest as possible. In fact, masterbatches are formulated to be dosed in the smallest possible proportion. The lower limit is often given by the capacity of industrial equipment to dose low quantities in a controlled manner. In general, the recycling industries have economic dosing equipment. For example, a dosage of $1 \%$ w/w, although desirable, would be very imprecise and difficult to control in a volumetric dispenser. That is why a value of commitment between price and reliability of the dosage was chosen in 3\% w/w, and a new blend PP-3MB4 was processed. Our results were good; since they show that only $3 \% \mathrm{w} / \mathrm{w}$ of MB4 induce similar benefits as $10 \% \mathrm{w} / \mathrm{w}$ (Table 1) with the same compatibilization level, and obviously reduced costs.

\section{Post-consumer recycled PP/LLDPE blends}

Since $3 \% \mathrm{w} / \mathrm{w}$ of MB4 showed to be an efficient compatibilizer for pristine blends, our aim was to verify this result in post-consumer recycled PP/LLDPE blends.

Morphology of PCR blends result similar to those of pristine blends (see Fig. 6), i.e. a salami type structure in which a polypropylene matrix with islands of polyethylene that contain occlusions of the matrix material is observed. Again, MB4 addition changes the morphology of the second phase from spherical shaped to elliptical or nondefined shaped domains $[\mathbf{2 3}, \mathbf{2 5}, \mathbf{2 6}, \mathbf{2 9}]$.

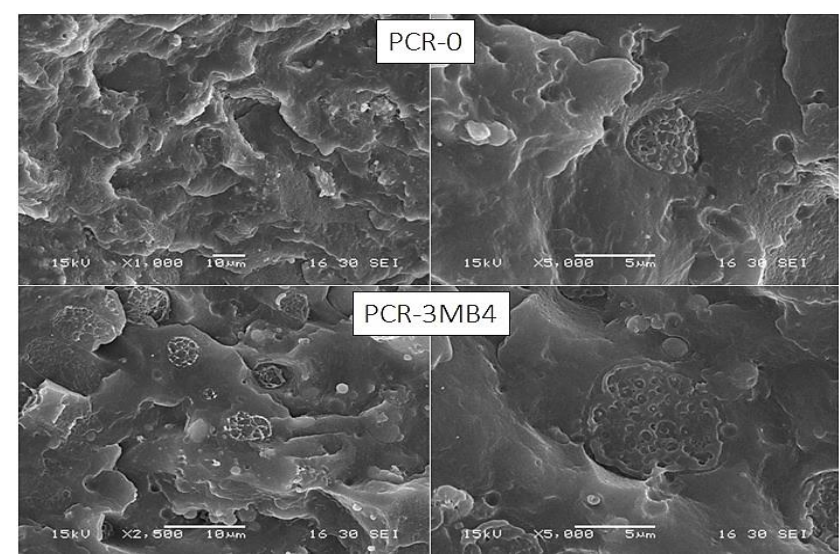

Fig. 6. Scanning electron micrographs of cryo-fractured surfaces showing the morphology of recycled blends (left: low magnification level, right: high magnification level): a) and b) PCR-0, c) and d) PCR-3MB4.
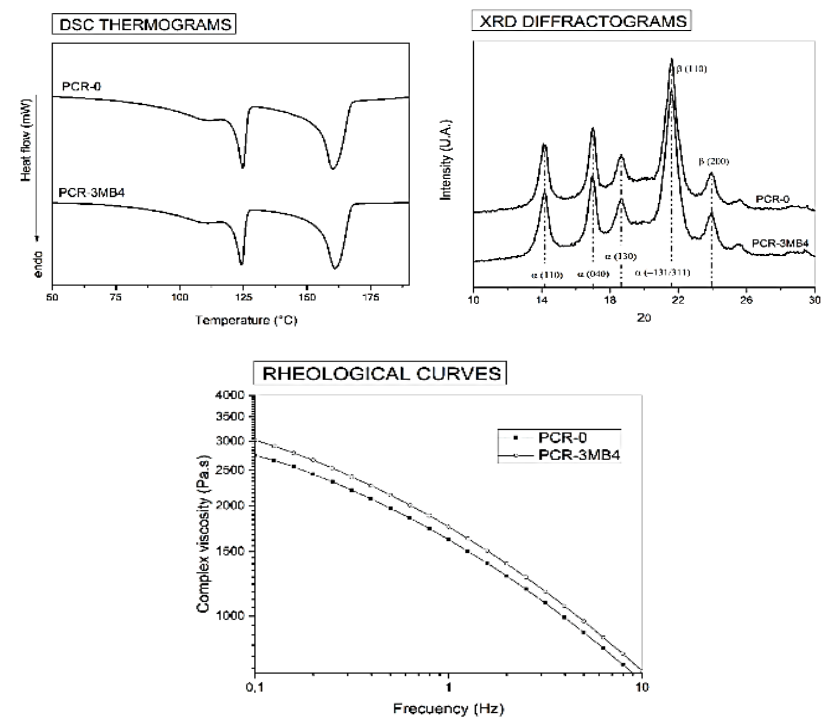

Fig. 7. Curves resulting from microstructural characterization of post consume recycled blends.

The last analysis indicates that recycled blends seem to be similar to pristine blends in terms of the above characteristics. That is, when a blend is compatibilized (either virgin o PCR), thermal and rheological behaviour is modified respect to uncompatibilized blend. Thus, results found for pristine blends are expected to be extrapolable to recycled ones, at least as a first approximation. To verify this hypothesis, the same mechanical and fracture characterization made for pristine blends was performed to PCR blends. MB4 presence improves both Young's modulus and tensile strength of the recycled blend (Table 3).

Table 3. Comparison of properties of recycled blends.

\begin{tabular}{cccccc}
\hline Sample & $\begin{array}{c}\text { Crystallinity } \\
(\boldsymbol{\%})\end{array}$ & $\begin{array}{c}\text { Young's } \\
\text { Modulus } \\
(\mathbf{M P a})\end{array}$ & $\begin{array}{c}\text { Yield } \\
\text { stress } \\
(\mathbf{M P a})\end{array}$ & $\begin{array}{c}\mathbf{J}_{\mathbf{m a x}} \\
\left(\mathbf{K J} / \mathbf{m}^{2}\right)\end{array}$ \\
\hline PCR-0 & $\begin{array}{c}\text { LLDPE } \\
\text { PP }\end{array}$ & $\begin{array}{c}28 \pm 4 \\
44\end{array}$ & $935 \pm 47$ & $17.8 \pm 0.8$ & $13.1 \pm 2.1$ \\
PCR-3MB4 & $\begin{array}{c}\text { LLDPE } \\
\text { PP }\end{array}$ & $\begin{array}{c}27 \pm 2 \\
40 \pm 4\end{array}$ & $1015 \pm 32$ & $18.5 \pm 0.4$ & $18.4 \pm 2.1$ \\
\hline
\end{tabular}

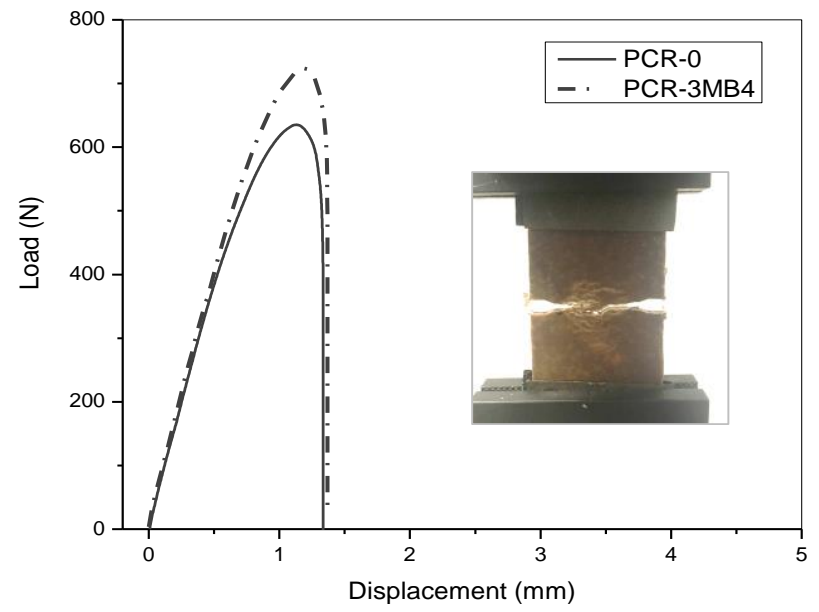

Fig. 8. Load-displacement curves from fracture tests along with macroscopic damage. 


\section{Advanced Materials Letters www.vbripress.com/aml}

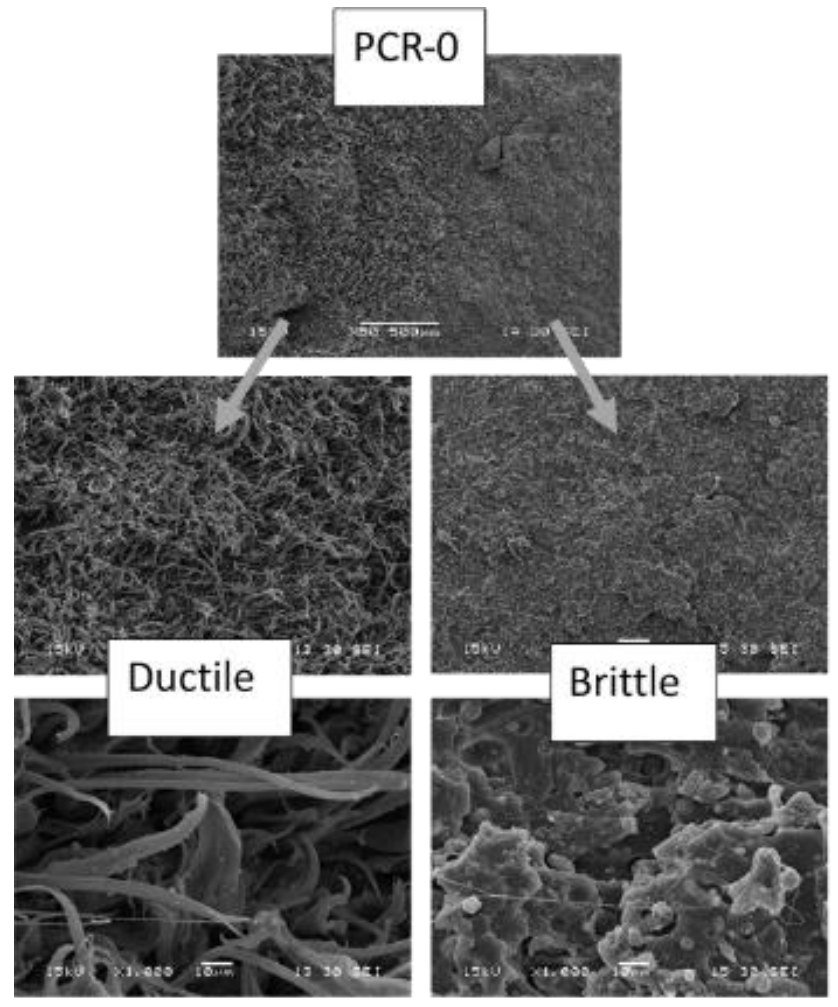

Fig. 9. SEM micrographs of fracture surfaces.

In fracture tests, an unstable brittle fracture after reaching the maximum load is seen, characterized by a slight non-linear load-displacement behavior (Fig. 8), compatible with the presence of rigid impurities $[\mathbf{1 8 , 3 3}$. Crack failure is also accompanied by the occurrence of secondary cracks [34]. Probably, these secondary cracks arose from impurities, which act as stress concentrators, promoting defects creation and a path for crack propagation. SEM micrographs of fracture surfaces also show a competition between two failure mechanisms: ductile, with fibrillation, and brittle, where fibrillation of the LLDPE domains is suppressed (Fig. 9). In addition, PCR-3MB4 also presents the highest matrix plastic deformation. When failure occurs in a ductile manner, is known to be due to develop and growth of voids at the second phase interfaces as a result of a decohesion process. In the opposite way, particles agglomeration hinders matrix fibrillation, inducing brittle failure. Then, it is easy to relate the macroscopic response to the blend's morphology described above. Moreover, $J_{\max }$ values show that PCR-3MB4 presents a higher crack initiation resistance than PCR-0 (Table 3), indicating that the presence of the compatibilizer not only affects morphology, but also improves phases adhesion inducing a better stress transfer.

\section{Conclusion}

In the present work structural, morphological, thermal, rheological, tensile and fracture characterization was performed on both virgin and post-consumer recycled PP/PE blends.

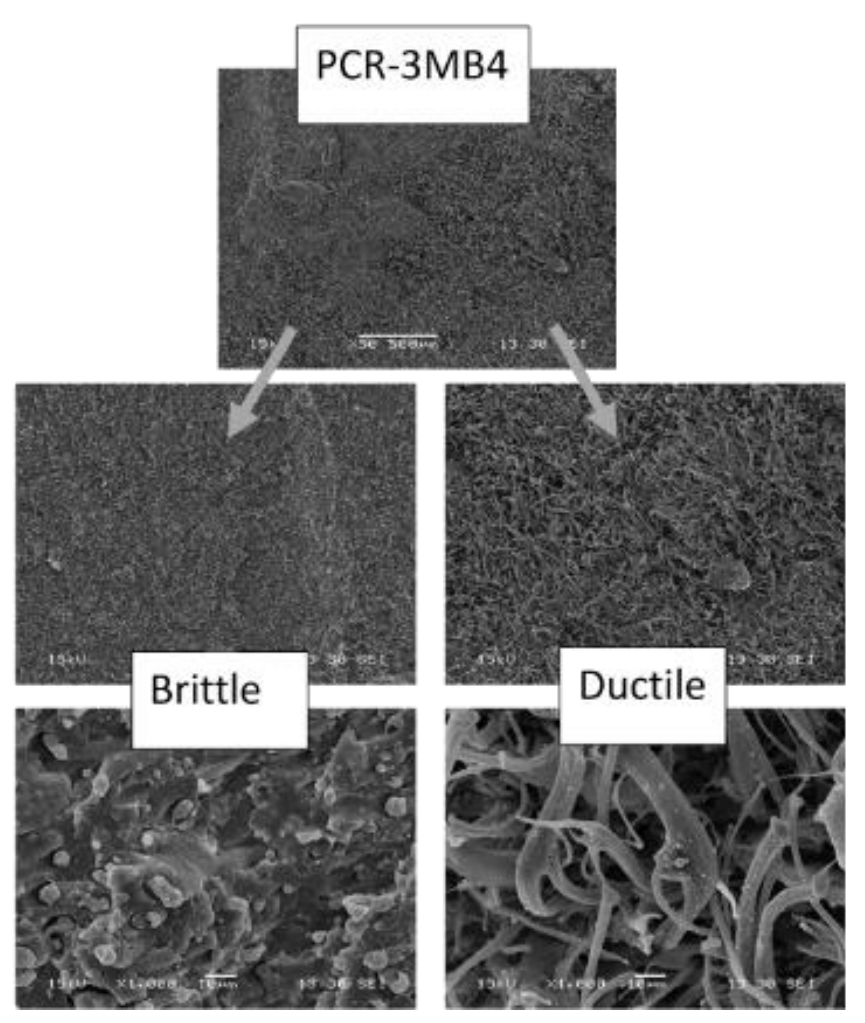

It was found that pristine and recycled blends present similar behaviour in terms of the evaluated characteristics, indicating that a study in virgin blends could be extrapolated to recycled blends at least as a first simple approach, in spite of the not complete known composition of PCR blends and the presence of impurities.

The effect of compatibilization was not possible to be detected from tensile tests. However, fracture behaviour was found to be sensitive to differences in microstructure induced by the presence of masterbatches in the blends, aiding in the selection of the best compatibilizer.

Blending with a suitable compatibilizer has been found effective in upgrading the performance of virgin and recyclates. The addition of only $3 \%$ of a commercial compatibilizer in a form of a masterbatch induces improvements in fracture behaviour and toughness, when compared with blends without compatibilizer, due to differences induced both in blend's morphology and in adhesion between phases.

\section{Acknowledgements}

Authors would like to thank ANPCyT, University of Mar del Plata (UNMdP) and University of Buenos Aires (UBA) for financial support.

Keywords

Recycled blends, polyolefins, compatibilizer, mechanical performance.

Received: 23 May 2020

Revised: 27 July 2020

Accepted: 03 September 2020 


\section{Advanced Materials Letters www.vbripress.com/aml}

\section{References}

1. Chiu, F.C.; Yen, H.Z.; Lee, C.E.; Polym. Test., 2010, 29, 397.

2. Dhoble, A.; Kulshreshtha, B.; Ramaswami, S.; Zumbrunnen, D.A.; Polymer, 2005, 46, 2244.

3. Jose, S.; Aprem, A.S.; Francis, B.; Chandy, M.C.; Werner, P.; Alstaedt, V.; Thomas, S.; Eur. Polym. J., 2004, 40, 2105.

4. Kallel, T.; Jaziri, M.; Massardier-Nageotte, V.; Gerard. J. F.; J. Appl. Polym. Sci., 2003, 90, 2475.

5. Noel, O.F.; Carley, J.F.; Polym. Eng. Sci., 1984, 24, 488.

6. Tai, C.M.; Li, R.K.Y.; Ng, C.N.; 1998, 19, 143.

7. Paolo, L.M.F.; Prog. Rubber, Plast. Recycl. Technol., 2004, 20, 11.

8. Jose, J.; George, S.M.; Thomas, S.; Network, 2011, 37, 187.

9. Teh, J.W.; Rudin, A.; Keung, J.C.; Adv. Polym. Technol., 1994, 13, 1.

10. Radonji, G.; Gubeljak, N.; Macromol. Mater. Eng., 2002, 287, 122.

11. Barlow, J.W.; Paul, D.R.; Polym. Eng. Sci., 1984, 24, 525.

12. Yang, M.; Wang, K.; Ye, L.; Mai, Y.-W.; Wu, J.; Plast. Rubber Compos., 2003, 32, 21.

13. Yang, M.; Wang, K.; Ye, L.; Mai, Y.-W.; Wu, J.; Plast. Rubber Compos., 2003, 32, 27.

14. Bertin, S.; Robin, J.; Eur. Polym. J., 2002, 38, 2255.

15. Fang, C.; Nie, L.; Liu, S.; Yu, R.; An, N.; Li, S.; Compos. Part B Eng., 2013, 55, 498.

16. Almaaded, M.A.; Madi, N.K.; Hodzic, A.; Soutis, C.; J. Therm. Anal. Calorim., 2014, 115, 811.

17. McCaffrey, Z.; Torres, L.; Flynn, S.; Cao, T.; Chiou, B.S.; Klamczynski, A.; Glenn, G.; Orts, W.; Ind. Crops Prod., 2018, 125, 425.

18. Anderson, T.; Fracture mechanics: Fundamentals and applications, 2017.

19. Yi, X.; Xu, L.; Wang, Y.L.; Zhong, G.J.; Ji, X.; Li, Z.M.; Eur. Polym. J., 2010, 46, 719.

20. Jayanarayanan, K.; Bhagawan, S.S.; Thomas, S.; Joseph, K.; Polym. Bull., 2008, 60, 525.

21. Fasce, L.A.; Frontini, P.M.; Wong, S.-C.; Mai, Y.-W.; J. Polym. Sci. Part B Polym. Phys., 2004, 42, 1075.

22. Sharma, R.; Socrate, S.; Polymer, 2009, 50, 3386.

23. Kunjappan, A.M.; Ramachandran, A.A.; Padmanabhan, M.; Mathew, L.P.; Thomas, S.; Macromol. Symp., 2018, 381, 1.

24. Mourad, A.-H.I.; Akkad, R.O.; Soliman, A.A.; Madkour, T.M.; Plast. Rubber Compos., 2010, 38, 265.

25. Penava, N. V.; Rek, V.; Houra, I.F.; J. Elastomers Plast., 2013, 45, 391.

26. Tselios, C.; Bikiaris, D.; Maslis, V.; Panayiotou, C.; Polymer, 1998, $39,6807$.

27. Harrats, C.; Thomas, S.; Groeninckx, G.; Micro- and Nanostructured Multiphase Polymer Blend Systems, 2006.

28. Ajitha A.R.; Thomas, S.; Introduction, Elsevier Inc., 2020.

29. Mourad, A.H.I.; Mater. Des., 2009, 31, 918.

30. Borovanska, I.; Dobreva, T.; Benavente, R.; Djoumaliisky, S.; Kotzev, G.; Elastomers Plast., 2012, 1-19.

31. Jin, M.; La, R.; Zhang, Y.; Liu, K.; Li, X.; Zhang, J.; Polym. Test., $\mathbf{2 0 1 5}, 42,135$.

32. Wunde, M.; Plagge, J.; Klüppel, M.; Eng. Fract. Mech., 2019, 214, 520 .

33. Iglesias Montes, M.L.; Cyras, V.P.; Manfredi, L.B.; Pettarín, V.; Fasce, L.A.; Polym. Test., 2020, 84, 106375.

34. Ho, M.; Wang, P.; Yeh, J.; 2016, 344-348. 